\title{
Uncovering critical cell death mechanisms for novel cardioprotective therapies
}

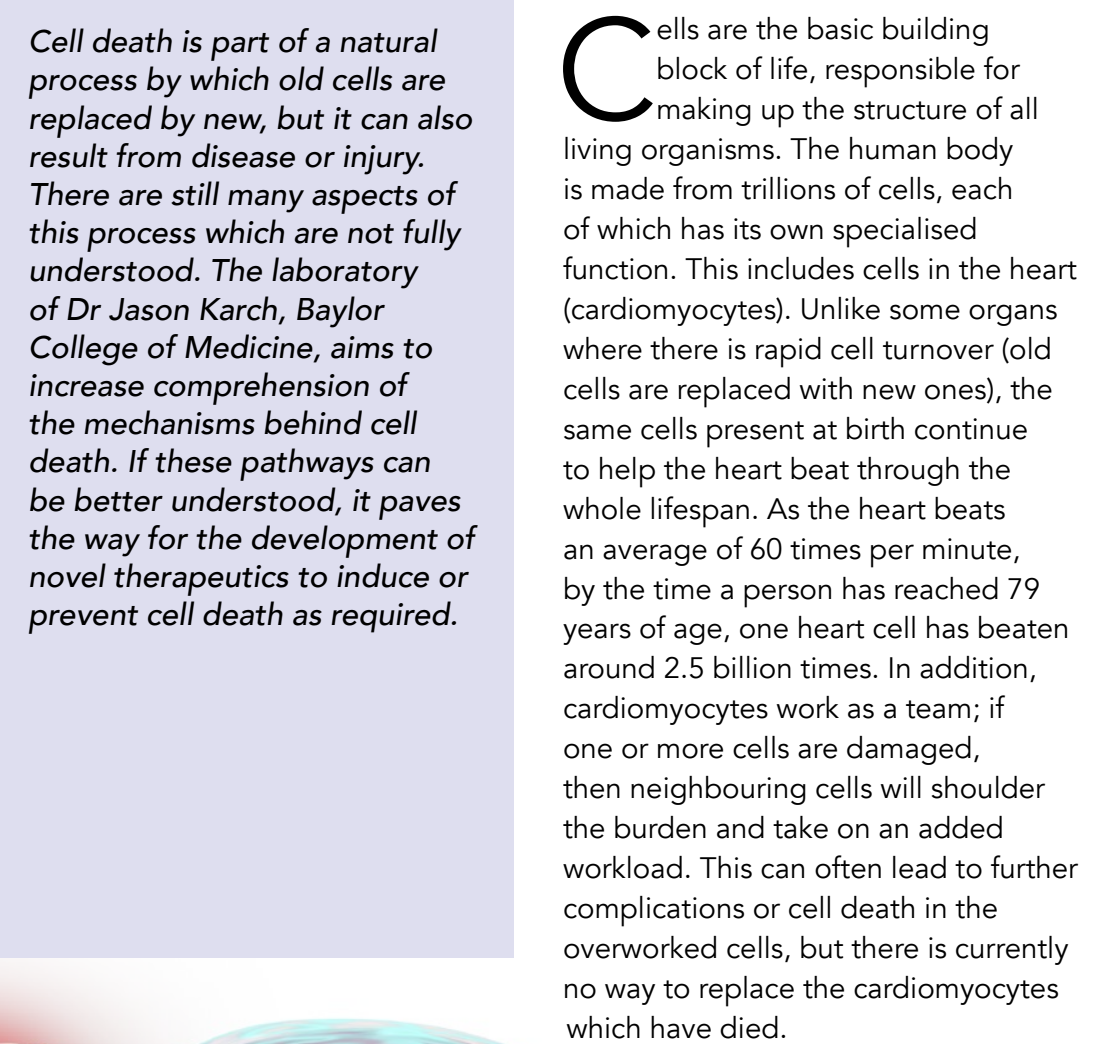

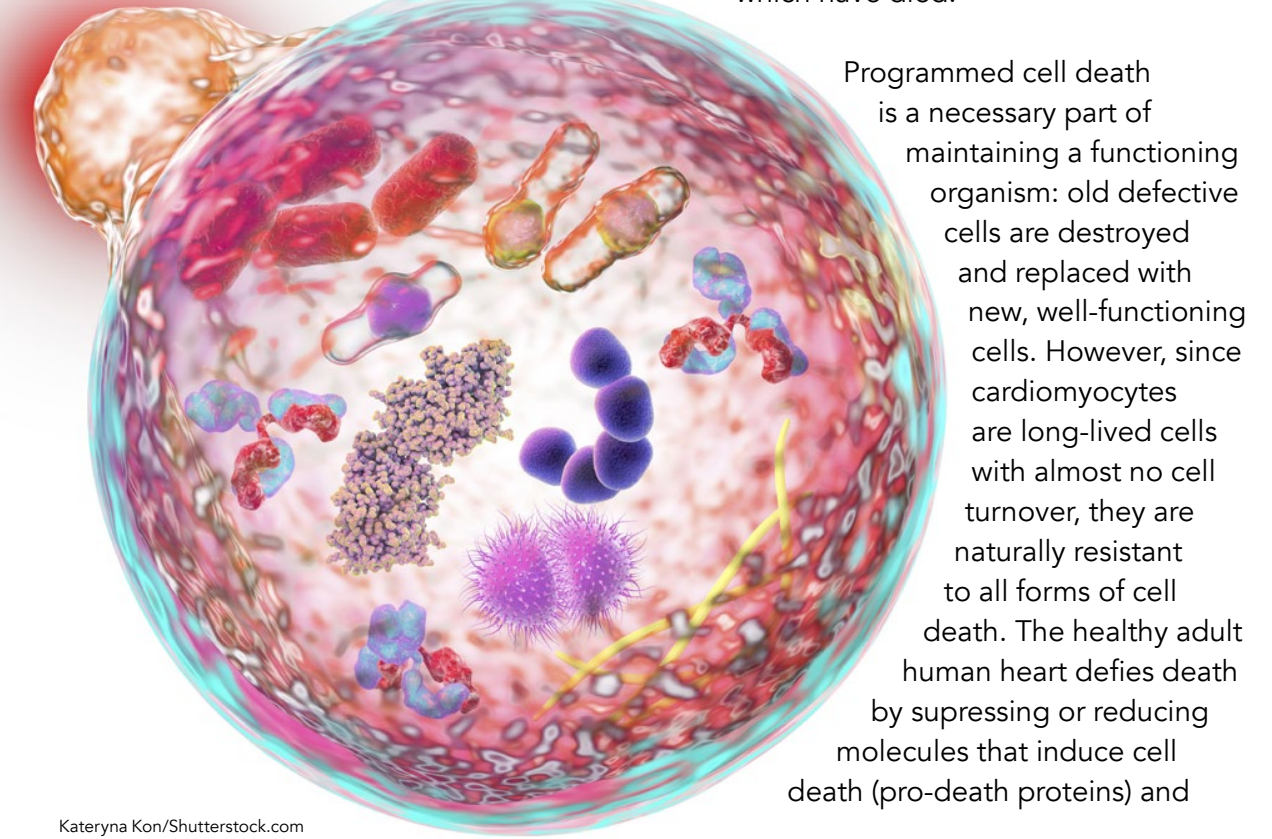

activating or increasing those that inhibit cell death (pro-survival proteins).

Changes in cell death processes epresent the underlying cause of many diseases, including cancer, wher when a lack of oxygen causes cells to die. Furthermore, loss of cardiomyocytes due to cell death is the underlying cause of most diseases of the heart, which is the leading cause of death in the developed world.

As the heart ages, changes occur which predispose the heart to many diseases, including arrhythmias, heart attacks and heart failure. It is estimated that in humans, around $30 \%$ of cardiomyocytes are lost between the ages of 20 and 75 years. This help explain why, along with accumulation of lifestyle-related changes, e.g. high we are more sugh blood pressure, we are more susceptible to cardic several mechanisms in place to try to educe these changes. For examplo autophagy is a heart-protective process that clears cellular debris and can delay the ageing process; it provides a 'housekeeping' service to keep the existing cardiomyocytes as healthy as possible. However, the autophagy process also decreases as we age.

We already know that, following a heart attack, the sooner the blockage causing the episode can be removed - and thus the oxygen supply to the heart ensured - the better. If this reperfusion process happens within heart muscle can be saved. The longer
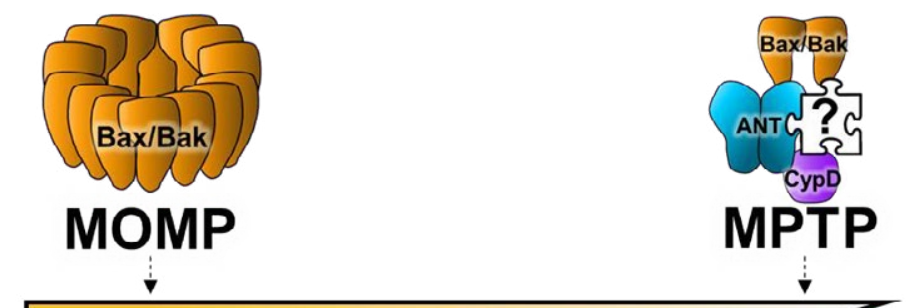

\section{Bax/Bak \\ Oligomerization}

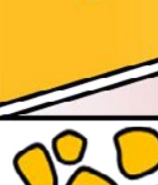

000

Apoptosis

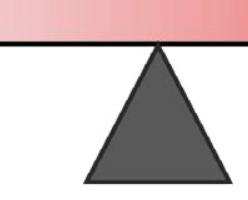

$\mathrm{Ca}^{2+} / \mathrm{ROS}$

\section{Inhibition of Bax/Bak offers a possible}

Since cardiomyocytes are long-lived cells with almost no cell turnover, they are naturally resistant to all forms of cell death.

that the blockage remains, the more
irreversible injury there is to the heart. irreversible injury there is to the heart.

MITOCHONDRIAL PORES

The work of Dr Jason Karch, Baylor

College of Medicine, focuses on

the pathways that are critical to

the execution or survival of cells,

particularly cardiomyocytes.

\section{CELLULAR POWERHOUSES}

Within each heart cell there are

plants called mitochondria. Mitochondria

are required to produce the vast amount

of energy that is required for the cell to beat. Cardiomyocytes, in particular, rely heavily on mitochondrial respiration as a source of energy. Thus, if significant amounts of mitochondria are damaged the cardiomyocyte will succumb to a process known as regulated necrosis (cell death).

A better understanding of how mitochondria become damaged will hopefully lead to new therapeutic approaches that prevent this damage times of stress This is a majorria in Dr Karch's research. tochondrial damage is a pore witin the membrane of the mitochondria referred to as the mitochondria permeability transition pore (MPTP) This is a pore that is formed in the inner certain conditions, such as following a stroke or heart attack. Upon the opening of this pore, the
mitochondrion becomes unable to produce energy and if it remains open for too long, the mitochondrion suffers irreversible damage. This is because the opening in the membran allows small molecules to enter the mitochondria and cause swelling. Both cell death through apoptosis (naturally occurring programmed cell death) or necrosis (premature cell death resulting from cell injury) can result from partial or prolonged MPT opening, respectively.

While modulation of the MPTP has been widely studied, the complete molecular mystery for decades, making some scientists question its existence.

In recent work, Dr Karch and colleagues genetically removed key components of this pore, which led to the complete inhibition of MPTP opening. The researchers focused on a protein, ANT, which is required to shuttle units of cellular energy (ATP)

out of the mitochondria. They found

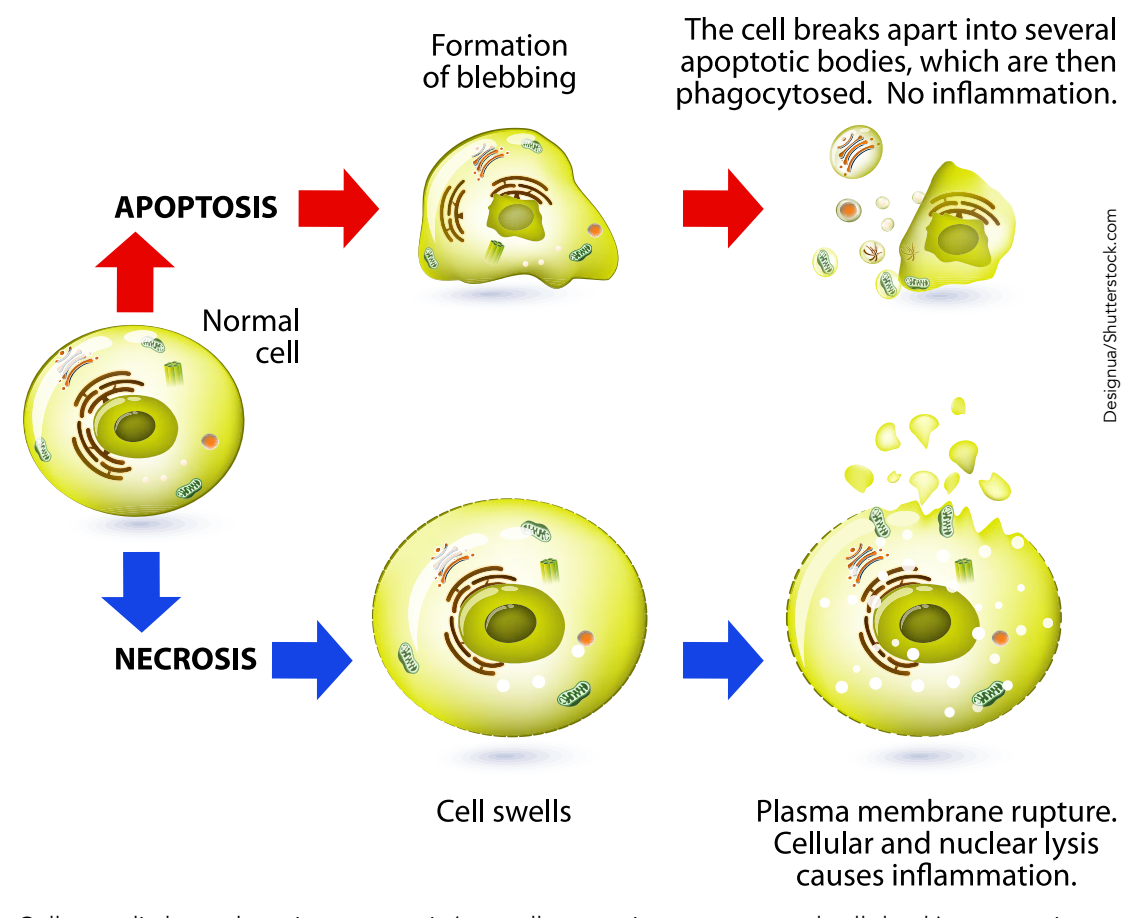

Cells can die by undergoing apoptosis (naturally
(premature cell death resulting from cell injury). 

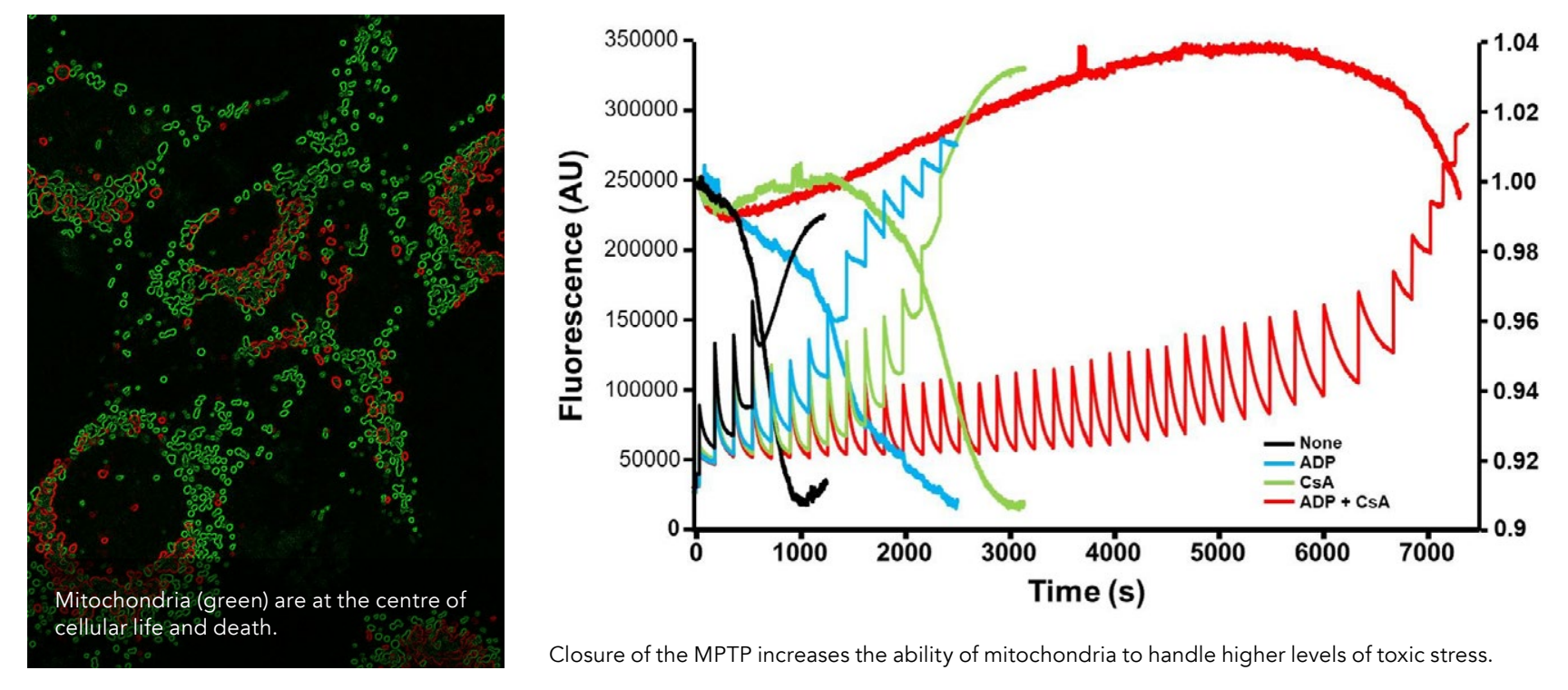

that mouse cells which were deficient in this protein showed a significantly Moreover. MPTP activity was also abolished, suggesting that no MPTP channels were present without ANTS. Indeed, ANT may be the primary pore-forming component of the MPTP. This work was carried out in mouse embryonic cells; therefore, much more work is required to translate this to the human body. However, it does shed light on the molecular composition of the MPTP.

In vivo, mitochondria lacking ANT are highly resistant to MPTP opening, suggesting a 'multi-pore model where the MPTP multiple distinct that this unknown sech surmises requires a protein called cyclophilin-D (CypD), as mice that lack CypD are also resistant to pore opening, and mice without ANTs and Cyp cannot undergo mitochondrial permeability transition. Interestingly, necrotic cell death is reduced in mice lacking CypD, while programmed cell death via apoptosis still occurs, This suggests that MPTP is primarily involved in aberrant pathological cell death rather than naturally occurring cell death. Further work is CypD-dependent component of the MPTP. This work that uncovered the one big step closer to finally identifying
the molecular components of the MPTP. $\quad \begin{aligned} & \text { components } \\ & \text { of the MPTP. }\end{aligned}$ permeability, Dr Karch's earlier work Once identified, it will be possible to explored two other proteins, Bax and design novel therapeutic approach researchers demonstrated that when to block MPTP opening during Bax/Bak were present on the outer mitochondria integrity and function, membrane of the mitochondria, MPTP- and protecting the cells of the heart dependent mitochondrial dysfunction from necrotic death. occurred normally. However, when the proteins were absent from the outer membrane, the permeability of the outer membrane was highly reduced, preventing MPTP-depende mitochondrial dysfunction and and colleagues concluded that either Bax or Bak mediate MPTP-dependent Buxture of the miloch and subs in these diseases as well. Thus, this work and its therapeutic applications subsequent cell death. Thus, Dr Karch
Furthermore, abnormal MPTP opening and necrotic cell death has diseases, such as Alzheimer's disease and muscular dystrophy. Accordingly, the MPTP is a desired clinical target cardiovascular system. been shown to contribute to other

\section{Be Behind the Research}

\section{Dr Jason Karch}

(2) E: karch@bcm.edu T: +17137982942

W: https://www.bcm.edu/research/labs-and-centers/faculty-labs/jason-karch-lab

\section{Research Objectives}

Dr Karch and his laboratory research the mechanisms behind cell death in order to better underta

\section{Detail}

Jason Karch

One Baylor Plaza

Houston, Texas 77030

USA

Bio

Dr Jason Karch is Assistant Professor at Baylor College of Medicine in the Department of Molecular Physiology and Biophysics and the Cardiovascular Research Institute. He has been a faculty member of Baylor College of Medicine since 2019 and was previously an Instructor at Cincinnati Children's ical Center.

Funding

- Aming

Blood Institute-National Institutes of Health

Collaborators

- Dr Jeffery Molkentin (Phd/Post-Doctoral Advisor) - Dr Pablo Piexoto (Collaborato)

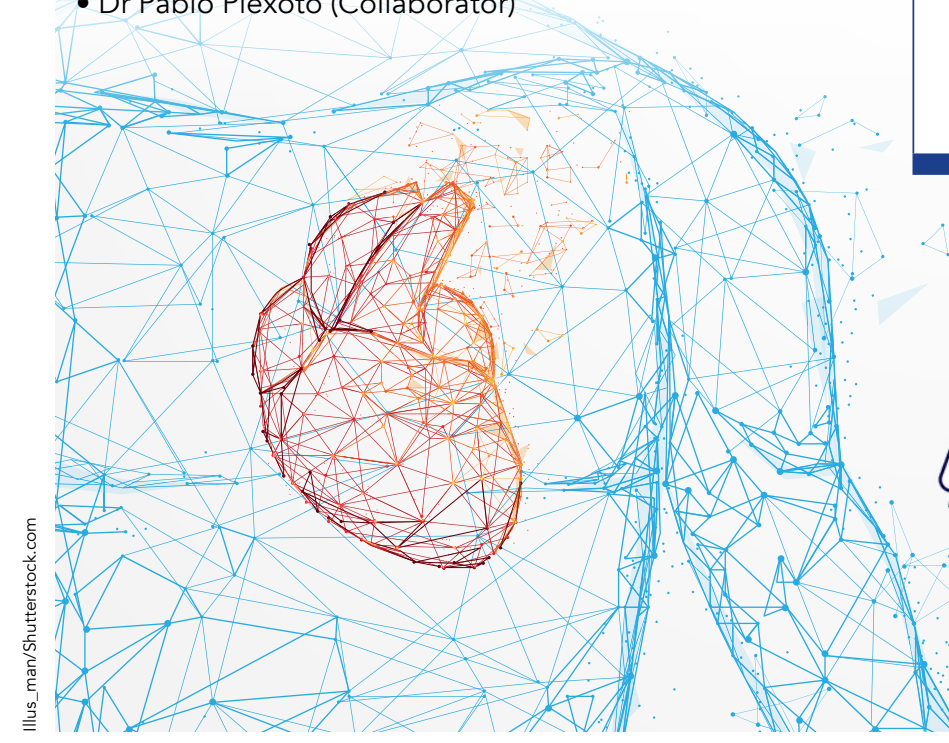

\section{References}

Karch, J., Bround, M. J., Khalil, H., Sargent, M. A., Latchman, N., Terada, N., Peixoto, P. M., and Molkentin, J. D. (2019). Inhibition of mitochondrial permeability transition by deletion of the ANT far ly and CypD. Science advances, 5(8), eaaw4597. Available at: https://dol. org/10.1126/sciadv.aaw4597

Karch, J., Kwong, J.Q., Burr, A.R., Sargent, M.A., Elrod, J.W., Peixoto, P.M., Martinez-Caballero, S., Osinska, H Cheng, E.H., Robbins, J., Kinnally, K.W., and Molkentin, J.D. (2013). Bax and Bak function as the outer membrane component of the mitochondrial permeability pore in necrotic cell death in mice. elife, 2, e00772. Available at: https://doi.org/10.7554/eLife.00772

\section{Personal Response}

What other cardiomyocyte-related projects are you working on currently?

II In the attempt to identify novel regulators of gain-of-function genome-wide screens during my post-doctoral training. As a result of these screening efforts, we identified numerous proteins as regulators of necrosis induced by calcium overload. Additionally, we identified a shorter list of proteins that, when added insult. We are currently defining the mechanisn of how these putative regulators protect or induce cell
death and their role in the heart.

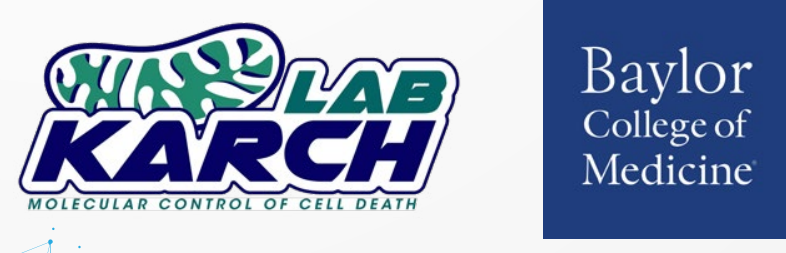

